\title{
Toward high-resolution global topography of Mercury from MESSENGER orbital stereo imaging: A prototype model for the H6 (Kuiper) quadrangle
}

\author{
Frank Preusker ${ }^{\mathrm{a}, *}$, Alexander Stark ${ }^{\mathrm{b}}$, Jürgen Oberst ${ }^{\mathrm{a}, \mathrm{b}, \mathrm{c}}$, Klaus-Dieter Matz ${ }^{\mathrm{a}}$, Klaus Gwinner ${ }^{\mathrm{a}}$, \\ Thomas Roatsch ${ }^{\mathrm{a}}$, Thomas R. Watters ${ }^{\mathrm{d}}$ \\ a German Aerospace Center, Institute of Planetary Research, D-12489 Berlin, Germany \\ b Technische Universität Berlin, Institute of Geodesy and Geoinformation Science, D-10623 Berlin, Germany \\ ${ }^{c}$ Moscow State University for Geodesy and Cartography, RU-105064 Moscow, Russia \\ ' Center for Earth and Planetary Studies, National Air and Space Museum, Smithsonian Institution, Washington, DC 20560-0315, USA
}

\section{A R T I C L E I N F O}

\section{Keywords:}

Mercury

MESSENGER

Stereo photogrammetry

Topography

Hun Kal

DTM

\begin{abstract}
A B S T R A C T
We selected approximately 10,500 narrow-angle camera (NAC) and wide-angle camera (WAC) images of Mercury acquired from orbit by MESSENGER's Mercury Dual Imaging System (MDIS) with an average resolution of $150 \mathrm{~m}$ /pixel to compute a digital terrain model (DTM) for the H6 (Kuiper) quadrangle, which extends from $22.5^{\circ} \mathrm{S}$ to $22.5^{\circ} \mathrm{N}$ and from $288.0^{\circ} \mathrm{E}$ to $360.0^{\circ} \mathrm{E}$. From the images, we identified about 21,100 stereo image combinations consisting of at least three images each. We applied sparse multi-image matching to derive approximately 250,000 tie-points representing 50,000 ground points. We used the tie-points to carry out a photogrammetric block adjustment, which improves the image pointing and the accuracy of the ground point positions in three dimensions from about $850 \mathrm{~m}$ to approximately $55 \mathrm{~m}$. We then applied high-density (pixel-bypixel) multi-image matching to derive about 45 billion tie-points. Benefitting from improved image pointing data achieved through photogrammetric block adjustment, we computed about 6.3 billion surface points. By interpolation, we generated a DTM with a lateral spacing of $221.7 \mathrm{~m} /$ pixel (192 pixels per degree) and a vertical accuracy of about $30 \mathrm{~m}$. The comparison of the DTM with Mercury Laser Altimeter (MLA) profiles obtained over four years of MESSENGER orbital operations reveals that the DTM is geometrically very rigid. It may be used as a reference to identify MLA outliers (e.g., when MLA operated at its ranging limit) or to map offsets of laser altimeter tracks, presumably caused by residual spacecraft orbit and attitude errors. After the relevant outlier removals and corrections, MLA profiles show excellent agreement with topographic profiles from H6, with a root mean square height difference of only $88 \mathrm{~m}$.
\end{abstract}

\section{Introduction}

Size, shape, and surface morphology constitute basic geodetic data for any planet. In March 2011, the MErcury Surface, Space ENvironment, GEochemistry, and Ranging (MESSENGER) spacecraft was inserted into orbit about Mercury (Solomon et al., 2001, 2008) and began a comprehensive mission. Several complementary techniques have been used to study Mercury's topography with MESSENGER data, including laser altimetry (Zuber et al., 2012), measurements of radio occultation times (Perry et al., 2015), limb profiling (Oberst et al., 2011; Elgner et al., 2014), and stereo imaging (Oberst et al., 2010; Preusker et al., 2011).

Although MLA on MESSENGER achieved a high single-shot ranging accuracy, MESSENGER's eccentric orbit and high northern periapsis restricted coverage to areas north of the equatorial region. Moreover, the spacing between orbital ranging tracks increased toward the equator, which reduced the areal density of the measurements at low latitudes. Hence, the ability to use laser measurements for morphological studies is limited.

The radio tracking of a spacecraft yields individual data points on the local radius of the target body when the spacecraft enters the radar shadow (ingress) or reappears (egress) (Fjeldbo et al., 1976; Perry et al., 2011, 2015). MESSENGER radio occultation measurements have been used to complement the limited MLA coverage to produce a global planetary shape model (Perry et al., 2015). From limb imaging one may determine topographic profiles along the planetary limb, which may be combined into planetary shape models (Dermott and Thomas, 1988; Thomas et al., 2007; Elgner et al., 2014). However, both radio occultation and limb profiling are hampered by the grazing viewing geometry and local variations in topography near the limb point.

\footnotetext{
* Corresponding author

E-mail address: frank.preusker@dlr.de (F. Preusker).
} 
Moreover, the models are insufficient for morphological studies because of their limited resolution.

Topographic models on regional scales were derived with stereo photogrammetry from MESSENGER images acquired during the mission's Mercury flybys (Oberst et al., 2010; Preusker et al., 2011). The goal of this paper, in contrast, is to move toward higher-resolution global models derived in a similar fashion from images acquired during MESSENGER's orbital phase. Such models require the use of higherresolution images and combinations of larger numbers of images as well as a carefully designed data processing strategy to maximize data product quality within the constraints of data processing resources.

In our processing, we use data from the most recent camera calibration effort (Oberst et al., 2011), which includes corrections for a temperature-dependent focal length shift. Also, as the images we use were obtained over the four years of the MESSENGER orbital mission phase (in contrast to the flyby images), updated Mercury rotation parameters have been used to correct the positioning of every image in the reference frame. To demonstrate and analyze the results of the processing of orbital images, we focus on a prototype digital terrain model (DTM), covering a single map quadrangle, the H6 (Kuiper) quadrangle.

\section{Initial data sets and requirements}

\subsection{The H6 quadrangle}

In order to manage the complexity and challenges of the global mapping task, we chose to derive individual regional terrain models following the planetary quadrangle scheme proposed for Mercury following the Mariner 10 mission (Greeley and Batson, 1990).

The H6 (Kuiper) equatorial quadrangle was chosen to demonstrate the production of a prototype. Like the other equatorial quadrangles, $\mathrm{H} 6$ extends from $22.5^{\circ} \mathrm{S}$ to $22.5^{\circ} \mathrm{N}$ latitude; $\mathrm{H} 6$ extends from $288.0^{\circ} \mathrm{E}$ to $360.0^{\circ} \mathrm{E}$ longitude. Hence, it includes crater Hun Kal $\left(0.5^{\circ} \mathrm{S}, 340^{\circ} \mathrm{E}\right)$, which defines Mercury's longitude system (Archinal et al., 2011). Inclusion of this feature affords us the opportunity to verify the correct alignment of our terrain model with the reference frame. An equatorial quadrangle was chosen, as these typically combine large numbers of WAC and NAC images, each requiring different sets of (temperaturedependent) calibration parameters. Also, comparisons with MLA tracks are possible that extend across the northern hemisphere to latitudes as far as $16^{\circ} \mathrm{S}$ (Section 6). H6 was also chosen because it demonstrates a particular challenge of the MESSENGER data - the "hot-season gaps" as discussed below (Section 2.2).

The H6 quadrangle hosts several prominent impact basins ( $>300 \mathrm{~km}$ in diameter), in addition to many large craters and tectonic features. The $\mathrm{H} 6$ area was also imaged by Mariner 10, and stereo topographic models derived from those images (Cook and Robinson, 2000) provide instructive comparisons (Section 7).

\subsection{MESSENGER orbit}

The MESSENGER spacecraft was in orbit about Mercury for slightly more than four years (from March 2011 to April 2015). During its first year in orbit, the spacecraft periapsis altitude was as low as $200 \mathrm{~km}$ at high northern latitudes, but the altitude was as great as $15,300 \mathrm{~km}$ at high southern latitudes with a spacecraft orbit period of $12 \mathrm{~h}$. In such a time interval the planet rotates by approximately $3.07^{\circ}$ and consecutive orbit tracks are consequently separated by $131 \mathrm{~km}$ near the equator. In April 2012 the orbit period was reduced to $8 \mathrm{~h}$, which reduced the separation of consecutive orbit tracks to approximately $2.05^{\circ}(\sim 87 \mathrm{~km}$ near the equator). Furthermore with the new orbit, the eccentricity was reduced, with the result that the spacecraft periapsis altitude was approximately $278 \mathrm{~km}$ and spacecraft apoapsis altitude moved to approximately $10,300 \mathrm{~km}$. Moreover, gaps in the mapping scheme emerged during "hot seasons" during which the spacecraft periapsis was over the dayside of Mercury, and all instruments had limited operations owing to thermal constraints.

\subsection{Laser altimetry}

The MLA performed laser pulse round-trip time of flight measurements to Mercury's surface with a repetition frequency of $8 \mathrm{~Hz}$ (Cavanaugh et al., 2007). Altimetry measurements are possible from ranges up to $1800 \mathrm{~km}$ with a single-shot ranging accuracy better than $1.0 \mathrm{~m}$ (Sun and Neumann, 2015). Owing to MESSENGER's eccentric orbit, the instrument could not range over most of the southern hemisphere. Furthermore, the attitude of the spacecraft was constrained by the orientation of the spacecraft sunshade to the Sun. Hence, instruments mounted on the spacecraft body, including MLA, were in off-nadir operation. This reduces the range distance capability and ranging accuracy of the instrument. However, at the lowest altitudes, MLA almost always had priority in spacecraft pointing and was nadir pointing. Owing to variations in spacecraft speed and ranging distance, laser footprint diameters and their spacing varied markedly over an orbit (Section 6).

\subsection{Camera system}

MESSENGER's MDIS consisted of two framing cameras, the WAC and the NAC, co-aligned on a pivot platform and equipped with identical $1024 \times 1024$-pixel charge-coupled device (CCD) sensors (Hawkins et al., 2007). The WAC featured 11 narrow-band filters from visible to near-infrared wavelengths and a broadband clear filter. In this paper, we used images taken by the WAC filter 7 (WAC-G), which was designed to have similar sensitivity to NAC filter $M$ with a maximum throughput at $750 \mathrm{~nm}$ (orange).

Both cameras consisted of a compact off-axis optical system that was geometrically calibrated with laboratory and in-flight data (Hawkins et al., 2007, 2009). The harsh thermal environment of Mercury requires sophisticated models for calibrations of focal length and distortion of the camera. In particular, the WAC camera and NAC camera were demonstrated to show a linear increase in focal length by $0.06-0.10 \%$ over the typical range of temperatures $\left(-20^{\circ} \mathrm{C}\right.$ to $\left.+20{ }^{\circ} \mathrm{C}\right)$ during operation, which causes a maximum displacement of $0.6-1.0$ pixels. Following methods described earlier (Oberst et al., 2011), the focal length dependence and geometric distortion for WAC and NAC were modeled using observations of star fields in different temperature regimes of the MESSENGER orbit.

\subsection{Stereo image coverage and image selection}

During the mission, MDIS acquired more than 200,000 images, most of them in the orbital mission phase. Owing to the spacecraft's eccentric orbit and high northern periapsis, an imaging strategy was chosen that combined the use of the WAC-G and NAC camera to cover both hemispheres at similar resolutions.

Note that with MESSENGER's maximum altitude at apoapsis, the lowest possible image resolution of NAC orbital images is about $320 \mathrm{~m} /$ pixel and about $295 \mathrm{~m}$ /pixel within the $\mathrm{H} 6$ quadrangle. In contrast, the highest image resolution of WAC images is about $100 \mathrm{~m} /$ pixel within the $\mathrm{H} 6$ quadrangle.

From image footprint information, we identified all narrow-angle and wide-angle filter $\mathrm{G}$ images that had a resolution between $50 \mathrm{~m}$ and $350 \mathrm{~m}$ that fell within the area of the H6 quadrangle. In total we found approximately 10,500 images, including about 8950 NAC images and about 1550 WAC-G images. The total included about 150 images from the second flyby in October 2008 (during which images were obtained occasionally from distances larger than $15,000 \mathrm{~km}$, Preusker et al., 2011).

The stereo-photogrammetric analyses require a favorable imageand illumination geometry, because such geometry affects the quantity 
Table 1

Optimal, adequate and minimal parameter ranges for key observing attributes. Note that the lower limit of the stereo angles is specified with two values indicating whether one pair to another pair within the set can have a lower minimum angle.

\begin{tabular}{llll}
\hline & Optimal & Adequate & Minimal \\
\hline Parameter & & & \\
Illumination variation & $0-10^{\circ}$ & $0-10^{\circ}$ & $0-10^{\circ}$ \\
Stereo angle & $15^{\circ}, 15-65^{\circ}$ & $5^{\circ}, 15-65^{\circ}$ & $5^{\circ}, 12-75^{\circ}$ \\
Incidence angle & $5-55^{\circ}$ & $5-80^{\circ}$ & $5-90^{\circ}$ \\
Emission angle & $0-55^{\circ}$ & $0-65^{\circ}$ & $0-70^{\circ}$ \\
Sun phase angle & $5-180^{\circ}$ & $5-180^{\circ}$ & $5-180^{\circ}$ \\
Stereo Coverage - Number of views (percent) & & \\
3 & 28.0 & 2.0 & 1.0 \\
4 & 22.5 & 3.6 & 1.2 \\
5 & 16.6 & 8.2 & 3.0 \\
$>5$ & 11.6 & 85.2 & 94.8 \\
Total & 78.7 & 99.0 & 100.0 \\
\hline
\end{tabular}

and quality of matched tie points (Section 4.2) and resulting DTM points. As image coverage, image scale, and illumination conditions varied substantially during the MESSENGER mapping mission, the "quality" of stereo conditions varied accordingly. We used data from our earlier MESSENGER image processing (Preusker et al., 2011) to define optimal, adequate, and minimal criteria for surface reconstruction (Table 1). We find "optimal stereo conditions" with at least threefold stereo coverage for about $79 \%$ of the area and "adequate stereo condition" for about $99 \%$ of the area within the H6 quadrangle. For the remaining areas, "minimal stereo conditions" apply. In the processing we began with areas having images at optimal stereo conditions, and we filled in remaining areas with images of adequate conditions first and finally minimal conditions (see Fig. 1).

To identify stereo combinations we formed a latitude-longitude grid of $0.1^{\circ} \times 0.1^{\circ}(720 \times 450$ grid elements). For each grid element, we identified the images covering the area (typically 10-300 images) and computed the stereo angles as well as the relevant illumination angles (i.e., Sun incidence, emission, and Sun phase angles) for each pair. Pairs were considered only if they had pixel scales that differed by less than or equal to a factor of three. All pairs were tested against the conditions of Table 1. We aimed to identify "combinations" of images concatenated by favorable stereo conditions. Typically, we found combinations with five to eight members; combinations with less than three images were discarded from the subsequent analysis.

Several groups of stereo image combinations extending over wide areas of the grid space were identified and were combined into "stereo networks." (Those groups were taken at similar local time during dedicated imaging campaigns.) Two large stereo networks, each containing several thousand images, were identified. Each network included images tied through favorable conditions but not sharing favorable conditions with images of the other network (see Fig. 2). Several smaller stereo networks, having fewer than 50 images, were removed from the analysis as they contributed little new data to the area.

The first main stereo network (see Fig. 2a) consists of about 15,300 stereo combinations including 5150 images (among 4500 NAC and 650 WAC-G images), taken at local afternoon time. The second smaller stereo network (see Fig. 2b) consists of about 5800 stereo combinations with about 2150 images (1600 NAC and 550 WAC-G images), taken in the local morning. Inspection reveals that these networks have similar Sun elevation angles of $20^{\circ}$ but nearly opposite Sun azimuth angles. To concatenate both networks, we manually created 135 threefold stereo combinations between images from the two networks (see Sections 4.2 and 5 for details).

In all we found about 21,235 independent stereo combinations consisting of about 7300 stereo images (6100 NAC and 1200 WAC-G images).

\section{Ancillary data}

We used nominal MESSENGER orbit and pointing data, as provided by the mission project, as well as the alignment of the camera to the spacecraft, and geometric calibration parameters of the camera (see NAIF PDS node). All ancillary data are typically provided in the form of SPICE kernels (Acton, 1996).

Furthermore, all computations were carried out in a Mercury-fixed

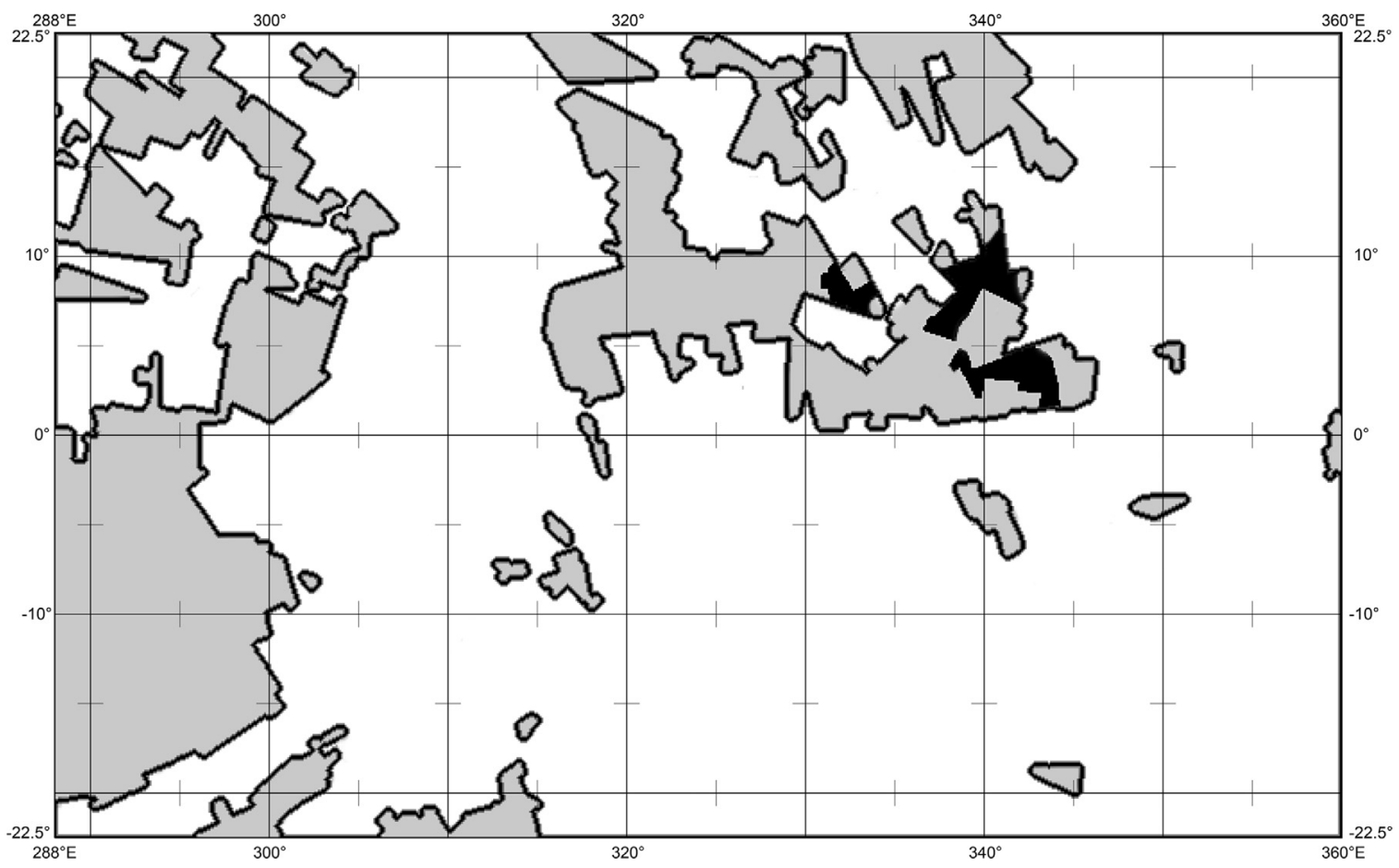

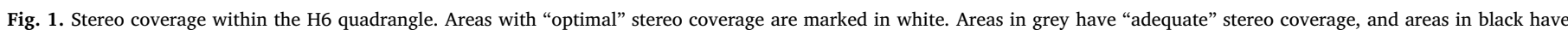
"minimal" stereo coverage (see text for further details). 

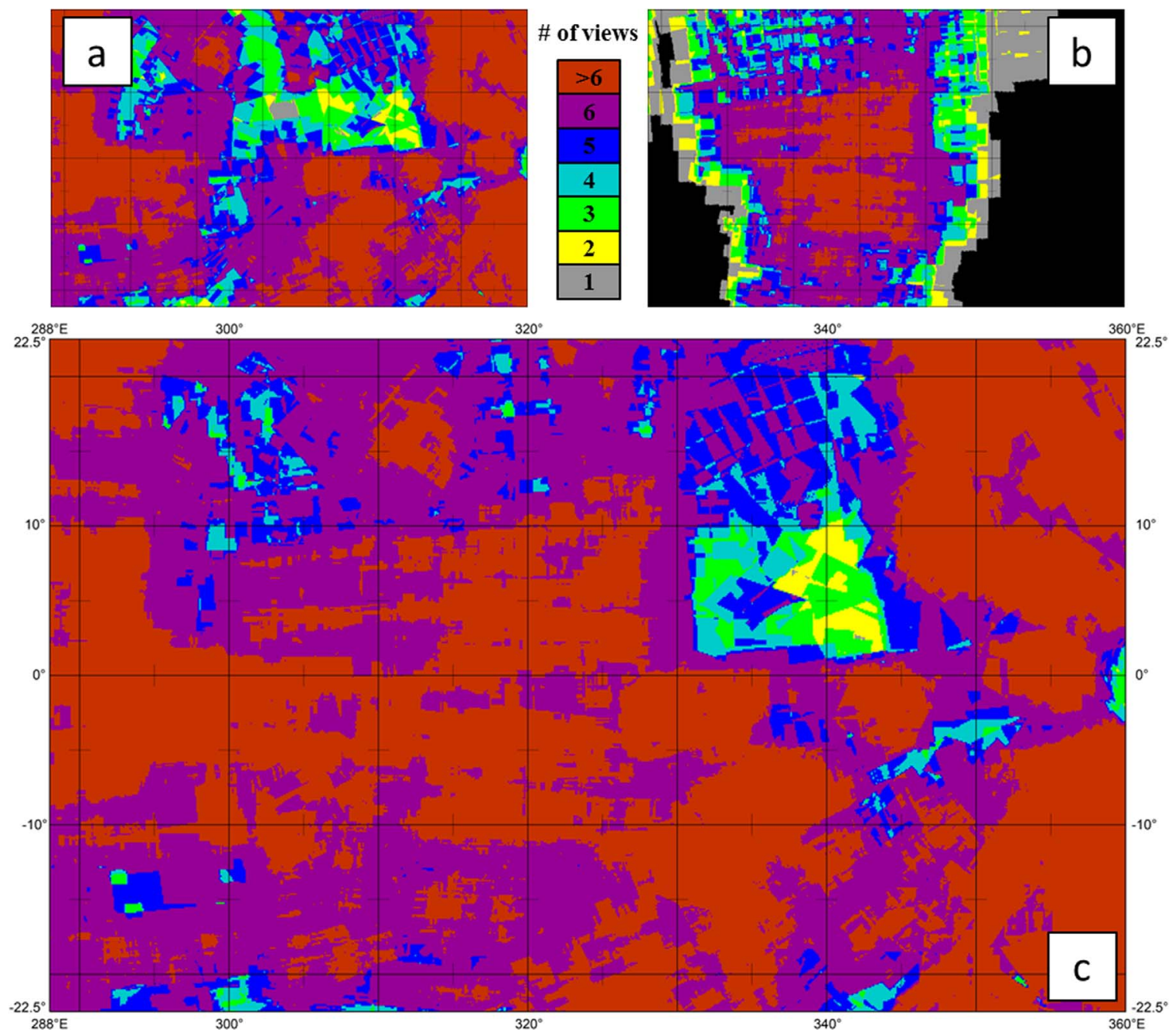

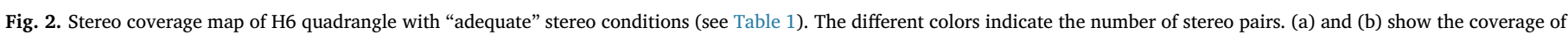

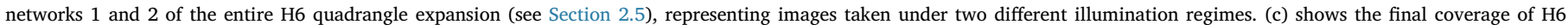
quadrangle after merging of the two networks.

reference frame, which was defined and agreed upon at an early stage within the MESSENGER science team (see pck00010_msgr_v23.tpc). This parameter set is a combination of libration and spin axis orientation estimates from Earth-based observations (Margot et al., 2012) and the rotation rate estimate from MESSENGER radio tracking data (Mazarico et al., 2014). While recently updated rotation parameters from co-registration of MESSENGER stereo images and laser altimeter data are available (Stark et al., 2015a), we used the "MESSENGER reference frame" to maintain consistency with other MESSENGER data products.

By combination of laser altimetry and radio occultation data, the mean radius of Mercury was derived as $2439.4 \mathrm{~km}$ (Perry et al., 2015). Although Mercury exhibits an ellipsoidal shape with axes of $\mathrm{a}=2440.5 \mathrm{~km}, \mathrm{~b}=2439.3 \mathrm{~km}$ and $\mathrm{c}=2438.3 \mathrm{~km}$ varying in the order of $1 \mathrm{~km}$, a sphere with the mean radius of $2439.4 \mathrm{~km}$ is used as a reference for our topographic model in this paper.

\section{Methods for DTM generation}

The construction of the DTMs followed procedures we used previously for Mercury (Oberst et al., 2010; Preusker et al., 2011, 2015). Those procedures can be broken down to five main tasks (Fig. 3).

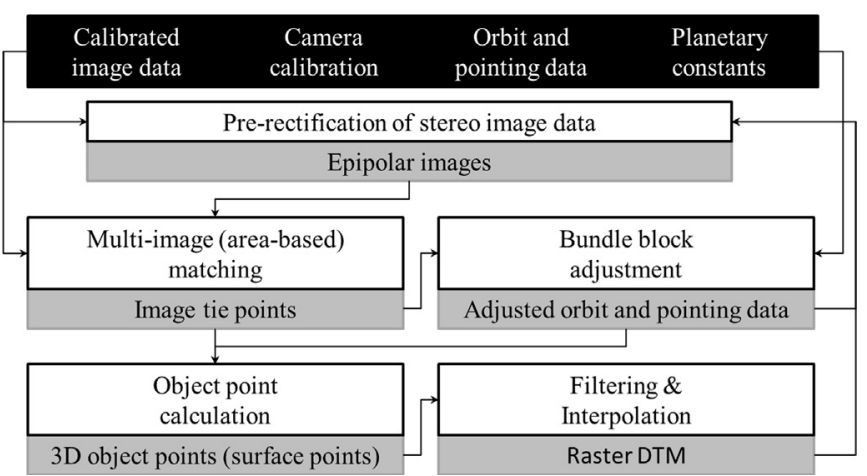

Fig. 3. Block diagram of the data processing sequence. Black boxes indicate input data, which enter the main five processing tasks described in Section 4. Grey boxes indicate resulting data.

\subsection{Pre-rectification}

From the nominal orbit and pointing information, all 7300 images were pre-rectified to a common map projection (here Lambert azimuthal projection), i.e., to one common scale, using Mercury's mean radius and a priori knowledge of the topography as reference. The processing was carried out in several stages following a pyramid strategy (Section 5), by which map scale and a priori topography for 

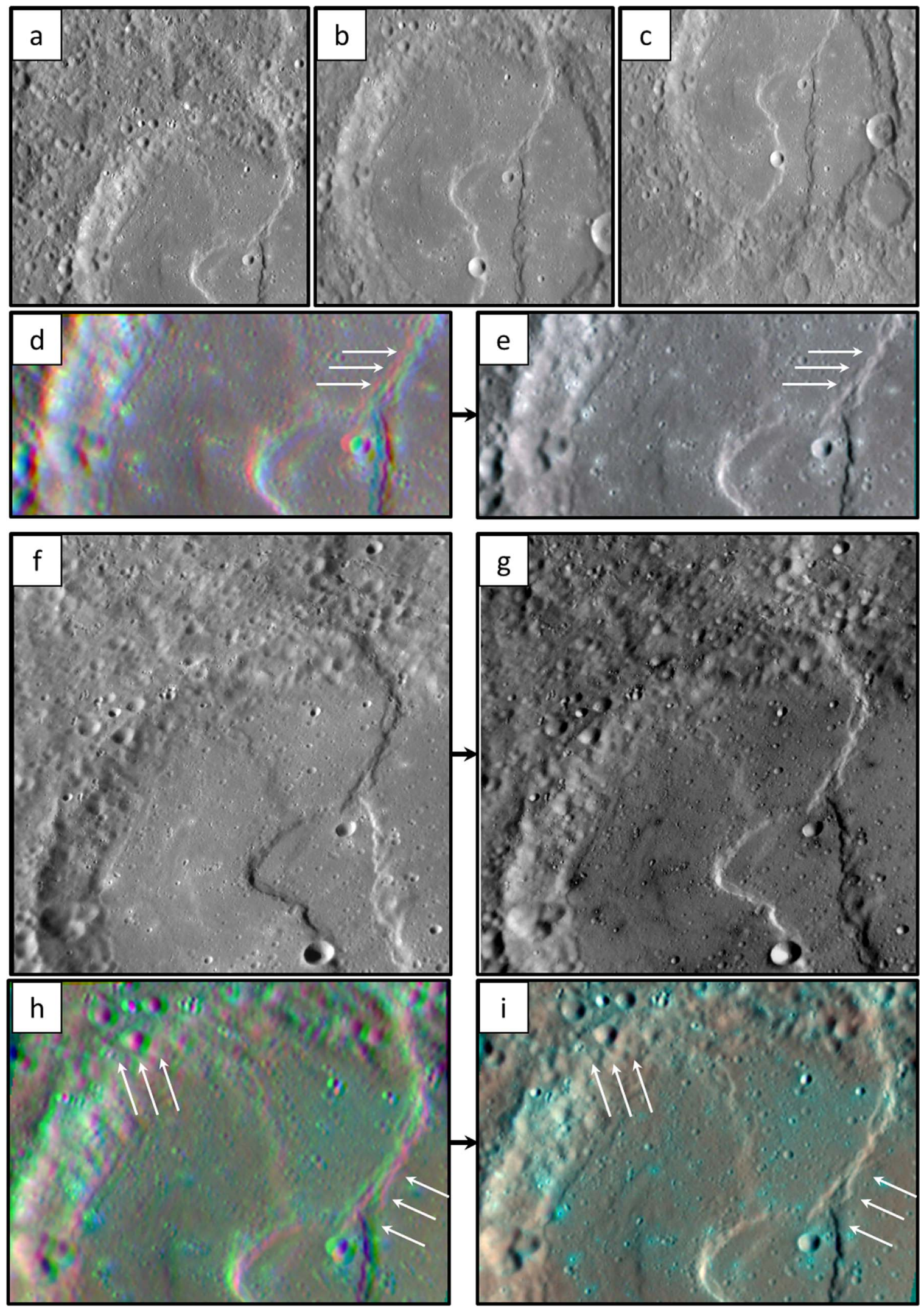

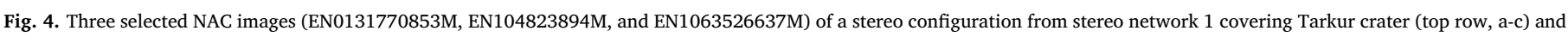

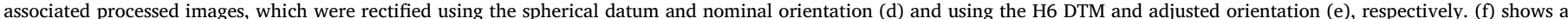

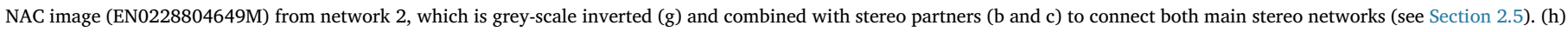

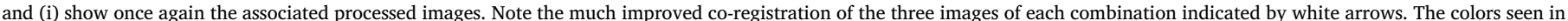
(d) and (h) show the misregistration of the images due to image pointing error, whereas the remaining colors in (i) are differences in reflectance due to grey-scale inversion of (f). 


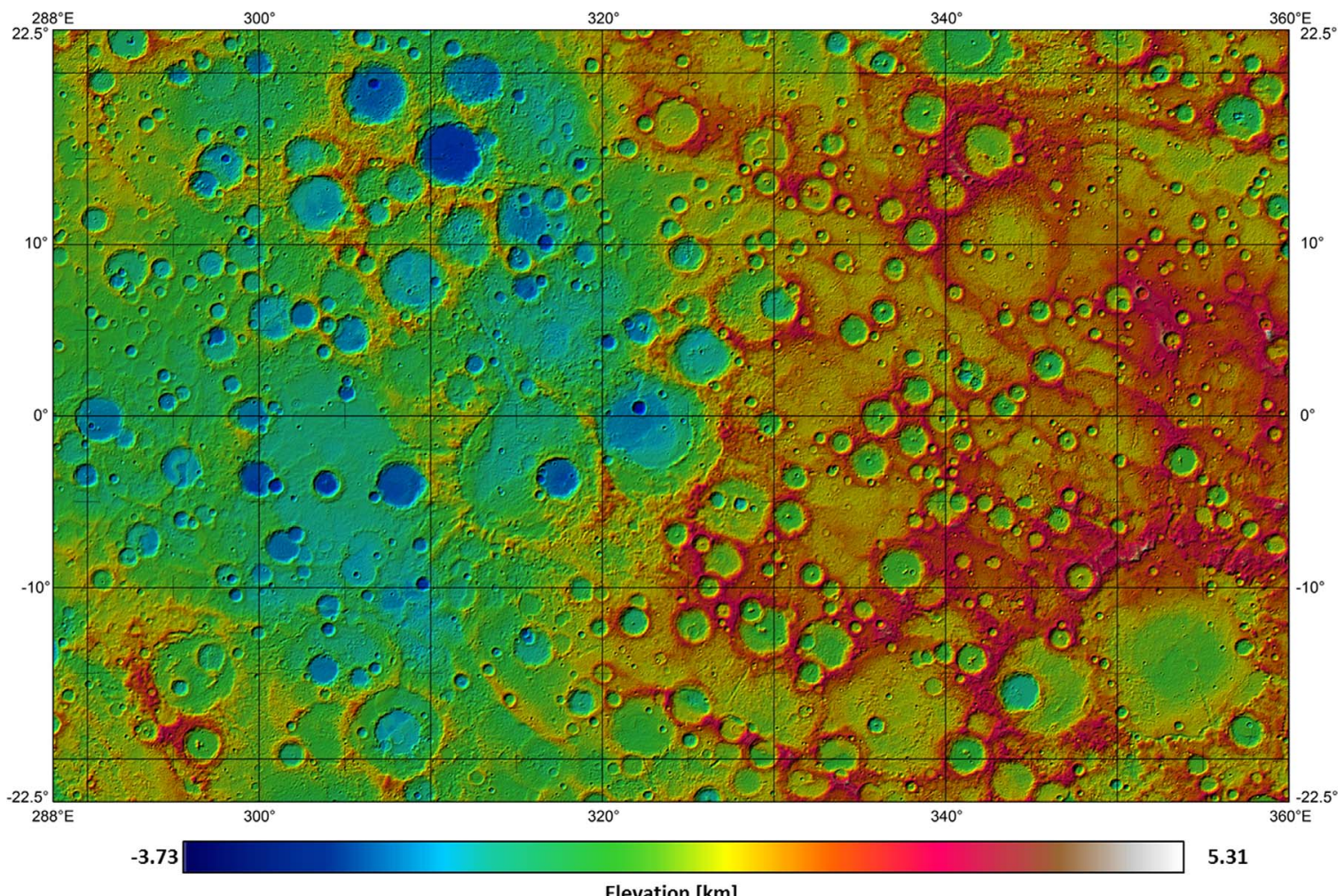

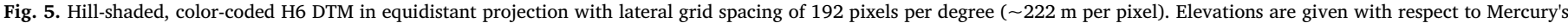
reference sphere of $2439.4 \mathrm{~km}$ radius.

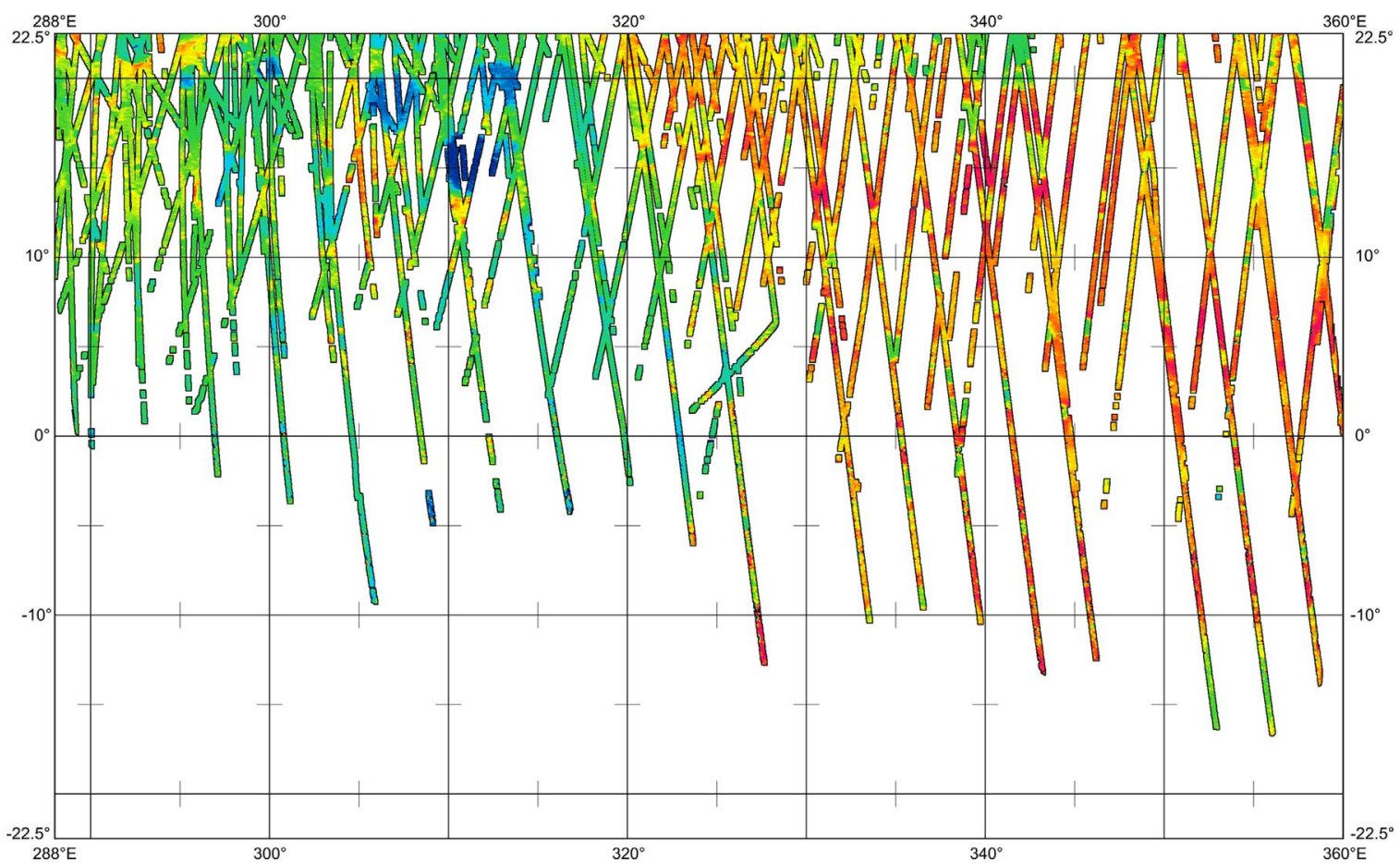

Fig. 6. MLA profiles within the same area as the H6 DTM. MLA heights are color-coded using the same color bar as in Fig. 5.

each pyramid level are selected accordingly.

This procedure was used to reduce search areas for multi-image matching (Section 4.2). Remaining image parallaxes reflect the deviation of the a priori topography assumption to the true shape of the body or inaccurate navigation data (see Fig. 4). In order to maintain the link of this pre-rectification geometry to the raw image geometry, the raw image coordinates for each pre-rectified pixel were stored in history files.

\subsection{Multi-image matching}

A multi-image matching technique (Wewel, 1996) was applied to the pre-rectified image data in order to derive conjugate points in each of the $\sim 21,000$ stereo combinations. The algorithm makes use of area- 


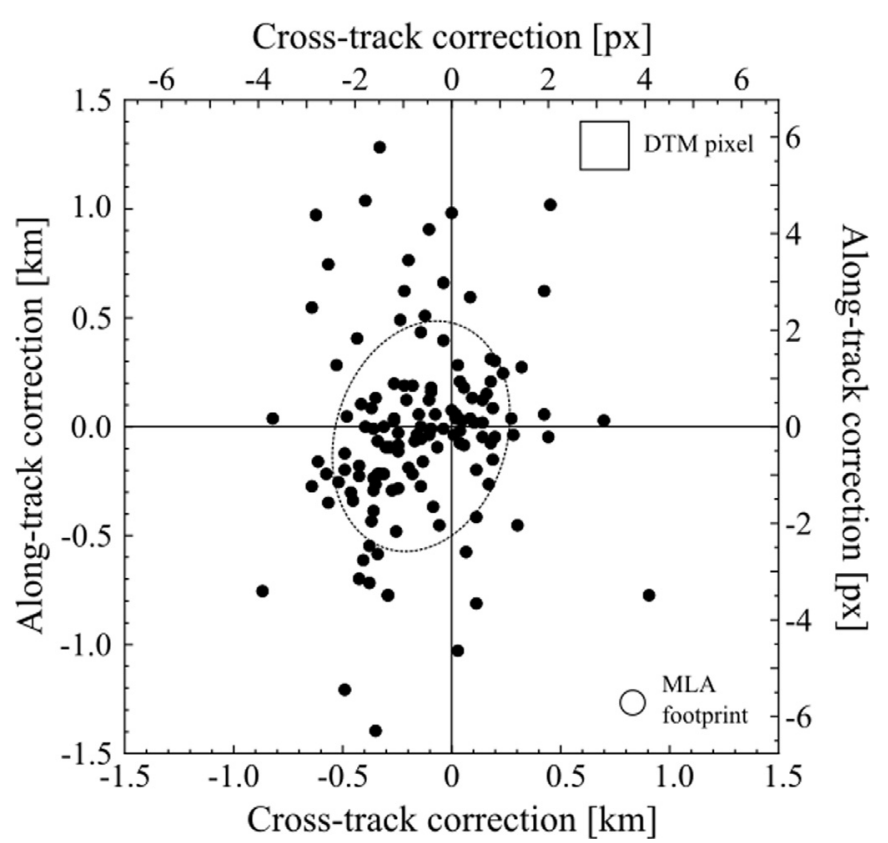

Fig. 7. Lateral (along-track and cross-track) corrections for MLA profiles after coregistration with the H6 DTM. The data scatter, which greatly exceeds the co-registration errors ( $<1 / 2$ pixel for most profiles), is thought to be due to spacecraft orbit and attitude uncertainties or residual errors of the Mercury rotation model. The dashed ellipses indicate the one-standard-deviation spread of the derived corrections. For comparison the size of a DTM pixel and the median diameter of all MLA footprints are shown.

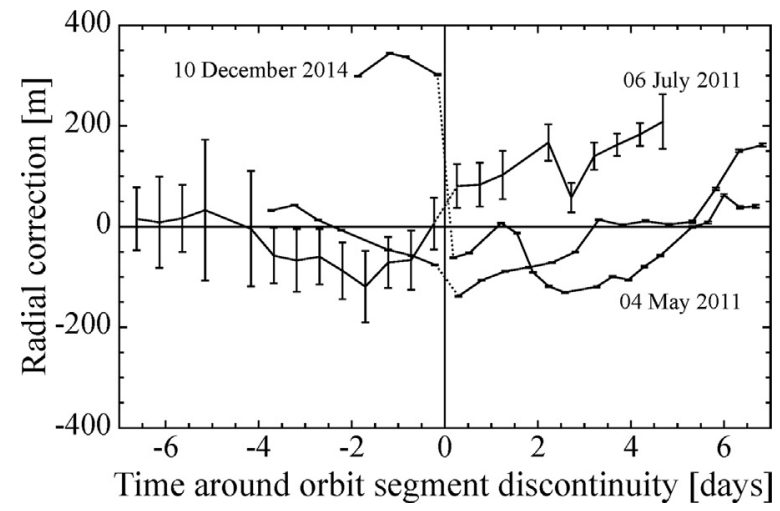

Fig. 8. Radial corrections for MLA profiles derived through co-registration to the H6 DTM. The labels denote the date of the orbit segment discontinuity event. All curves are centered with respect to their orbit segment discontinuity events. Error bars denote the uncertainty in the radial correction for the vertical co-registration of the respective MLA profiles. See Section 6 for further details.

based correlation to derive approximate values for the image coordinates, which are refined to sub-pixel accuracy by least-squares matching. The correlation was done for each master image as the reference image with all stereo partners, i.e., all overlapping images. After the multi-image matching process, the derived image coordinates were transformed back to raw data geometry, using the history files generated during the pre-rectification. The accuracy of this backtransformation is better than one tenth of a pixel (Scholten et al., 2005).

For each stereo combination, two kinds of tie-point data sets were generated. First, the images were matched in a sparse grid, usually every 20th pixel, whereas in a second run a higher-density grid was generated. Here, every pixel of the master image was matched with its stereo partners. The lower-density image point measurements (matched points) were the initial input for the bundle adjustment (Section 4.3) whereas the high-density measurements are used for DTM generation (Section 4.4).

Particular care was taken to match the 240 selected images combining the two stereo networks with their markedly differing illumination (Section 2.5). To enable the automatic matching, negative versions of the images from the second network were produced by inversion of pixel digital number (DN) values. These negative images were successfully matched with the images from the first network (Fig. 4).

\subsection{Bundle block adjustment}

The central software element is the bundle block adjustment, with which we carry out a least-squares inversion of image tie-point measurements to determine the six unknown camera orientation parameters (three metric parameters for the camera position and three angular parameters for the camera pointing) for every image as well as three coordinates for each tie-point in object space. The relation between tie-point coordinates and the corresponding surface point is mathematically defined through what are termed the collinearity equations (Albertz and Wiggenhagen, 2009).

Nominal navigation and reference frame data (Section 3) were used to begin the iterations. No attempt was made to further improve model parameters, e.g., planet rotation model, spacecraft orbit parameters, or camera constants, at this stage. We assumed that any systematic error would be spread over the adjusted navigation data of all images and would not affect the internal rigidity of the model.

\subsection{Object point calculation}

The line of sight for each observation, defined by the image coordinates, the geometric calibration, and the orientation data, was computed. Lines of sight for each tie-point were combined to compute forward ray intersections using least-squares techniques. We obtained object points in Cartesian coordinates and their relative accuracies. Again, the redundancy given by multi-stereo capability allowed us to accept only those object points that are defined by at least three stereo observations. Thus, we avoided occasional gross matching errors, typical for simple two-image matching.

\subsection{DTM interpolation}

For the generation of the gridded DTM we combined the object points of all models. First, all object points were transformed from Cartesian- to spherical coordinates (latitude, longitude, and radius). Height values were computed with respect to Mercury's adopted mean radius of $2439.4 \mathrm{~km}$. The latitude-longitude coordinates were transformed to the standard map projection of our quadrangle (here equidistant projection). Object points located within a DTM pixel were combined by a distance-weighted mean filtering technique (Gwinner et al., 2009), which also involves neighborhood data within a four-pixel radius. Finally for regions that lacked any object point information (mostly due to cast shadows), we applied a gap-filling algorithm using data from preceding DTM pyramid levels of reduced resolution in order to derive a raster DTM without gaps. The proportion of gaps with respect to the entire DTM was less than $0.25 \%$.

\section{Results}

The five steps described above were carried out at three levels, following a pyramid strategy, whereby reduced map scales were chosen for pre-rectification of the images (Section 4.1) at each step. DTMs produced in the first and second pyramid level and associated adjusted navigation data were used as a priori models for pre-rectification of images in the respective subsequent levels. We applied data snooping techniques within bundle block adjustment (see Section 4.3) to eliminate outliers (identified by large ray intersection errors) at each pyramid level. As a result of this strategy we obtained two sets of tiepoint observations for each stereo combination. Here, the count of tie- 


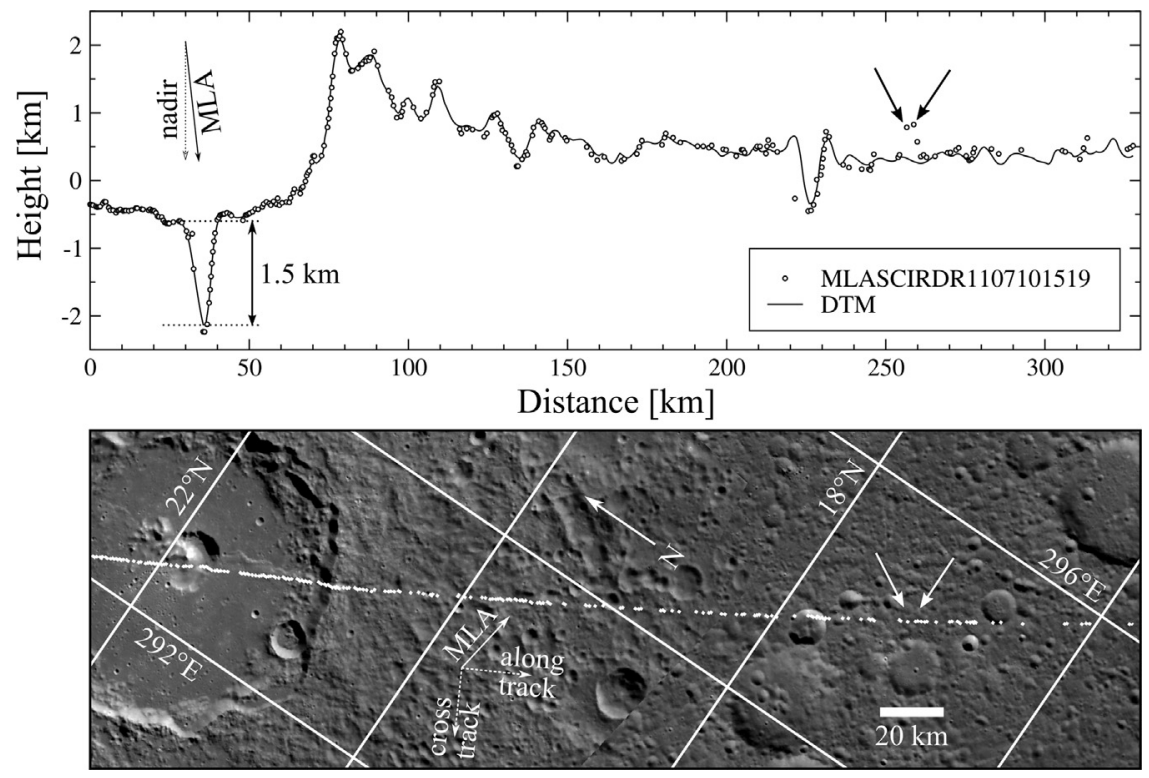

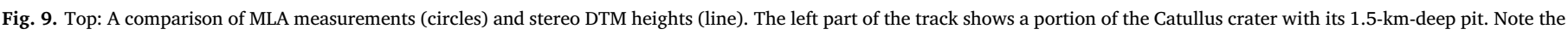

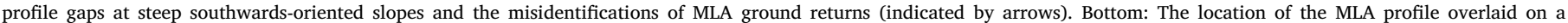
MESSENGER MDIS mosaic. The MLA measurements of this portion of the laser profile were performed at an off-nadir pointing of $35^{\circ}$.

points per stereo combination (depending on overlapping areas) for high-density matching ranged from about 200,000 to 4 million tie points and from 200 to 2000 for coarse matching. The resulting local DTMs and rectified images were used for quality assessment.

At the final block adjustment stage, we used a set of 250,000 tie point observations from coarse matching of 7800 images, which represent 50,000 individual ground points. Hence, the ratio between observations (545,000 image coordinates) and unknowns $(150,000$ ground point coordinates) is about 3.6. As a result of the adjustment, the mean accuracy of the ground points is reduced from $850 \mathrm{~m}$ (using nominal navigation data) to $55 \mathrm{~m}$. On average, the position and the pointing of the images were adjusted by $80 \mathrm{~m}$ (mostly in the radial direction) and $0.01^{\circ}$, respectively.

From our set of 21,000 stereo configurations, we derived coordinates for 6.3 billion ground points. For the gridded DTM, we set a grid spacing of 192 pixels per degree, corresponding to $221.7 \mathrm{~m} /$ pixel (see Fig. 5). Hence, we have 50 ground points within any DTM pixel, on average. The grid spacing was selected to reflect the mean resolution of all involved images (150 m/pixel). With the given coverage of approximately 5.9 million square kilometers and the given equirectangular projection, the H6 DTM has 8641 lines (rows) and 13,825 samples (columns).

The mean elevation level of the DTM is $0.88 \mathrm{~km}$ with respect to the reference Mercury radius, with heights ranging from $-3.73 \mathrm{~km}$ to $5.31 \mathrm{~km}$ and with $95 \%$ (two standard deviations) of the area between $-1.4 \mathrm{~km}$ and $2.9 \mathrm{~km}$. Visual inspection reveals that the terrain is characterized by an elevated area in the east and low areas in the west. The highest point within $\mathrm{H} 6$ is located at $4.8 \mathrm{~N}^{\circ}, 358.9 \mathrm{E}^{\circ}$, whereas the lowest point is located at $0.4 \mathrm{~N}^{\circ}, 322.2 \mathrm{E}^{\circ}$, associated with a deep crater within the Homer basin.

\section{Comparison with laser altimetry}

In the equatorial regions coverage by laser profiles is sparse (Fig. 6). Only 131 profiles (counting only those with more than 100 laser footprints) cover the area of the H6 DTM. The southernmost laser footprint is located at $16.727^{\circ} \mathrm{S}, \quad 356.050^{\circ} \mathrm{E}$ (in MLASCIRDR1105020655). Ranging was performed over distances varying from 200 to $1800 \mathrm{~km}$. Consequently, footprint diameters varied from 35 to $280 \mathrm{~m}$ (median of $117 \mathrm{~m}$ ). The footprint-to-footprint distance, driven by the ground-track velocity of the spacecraft, varied from 175 to $420 \mathrm{~m}$ (median of $310 \mathrm{~m}$ ), assuming that no laser returns were missed. The ranging accuracy of MLA, depending on distance and the incidence angle of the laser pulse on the surface, varied between 0.12 (for nadir observations) and $1.5 \mathrm{~m}$ (Sun and Neumann, 2015).

In order to compare the H6 DTM and the MLA profiles, a coregistration was performed following techniques described by Gläser et al. (2013) and Stark et al. (2015b). The optimum lateral and vertical positions of a laser profile with respect to the DTM were computed by minimizing the height differences between the data sets. For most profiles, the positions can be determined with accuracy better than the size of one DTM grid element. We computed offsets of the 131 MLA profiles with respect to their nominal positions, including the associated uncertainties.

Most lateral along- and across -track offsets (Fig. 7) are significantly larger than these uncertainties. The one-standard-deviation ellipse of the corrections has principal axes of 393 and $540 \mathrm{~m}$, and the center of the ellipse is offset by -143 and $-45 \mathrm{~m}$ in cross- and along track directions, respectively. We argue that this large spreading cannot be caused by internal geometric distortions of the DTM, but is more likely to be due to errors in spacecraft orbit and attitude uncertainties or residual errors in the Mercury rotation model.

Interestingly, the radial corrections for consecutive orbits follow some systematic trends (Fig. 8), with pronounced discontinuities, probably relics from the MESSENGER orbit determination process. The discontinuities are found to match with well-known "orbit segment discontinuities" (OSDs) ${ }^{1}$ (Fig. 8), originating from the fact that orbit determination was performed in discrete time segments of one week each, with parameters of the orbit determination force model estimated separately for each week (Page et al., 2014). In one particular case, the MLA profile prior to the OSD on 12 October 2014 is offset from a profile acquired after that OSD by more than $300 \mathrm{~m}$, which is much larger than the uncertainty in the co-registration or the height accuracy of the DTM. Note that the radial corrections, as opposed to the lateral corrections, are not as much influenced by the uncertainty in pointing but rather by uncertainty in the orbit. Furthermore, offsets may also be due to small residual errors in the Mercury rotation model.

\footnotetext{
${ }^{1}$ See comments in http://naif.jpl.nasa.gov/pub/naif/pds/data/mess-e_v_h-spice-6-v1. 0/messsp_1000/data/spk/msgr_040803_150430_150430_od431sc_2.bsp.
} 

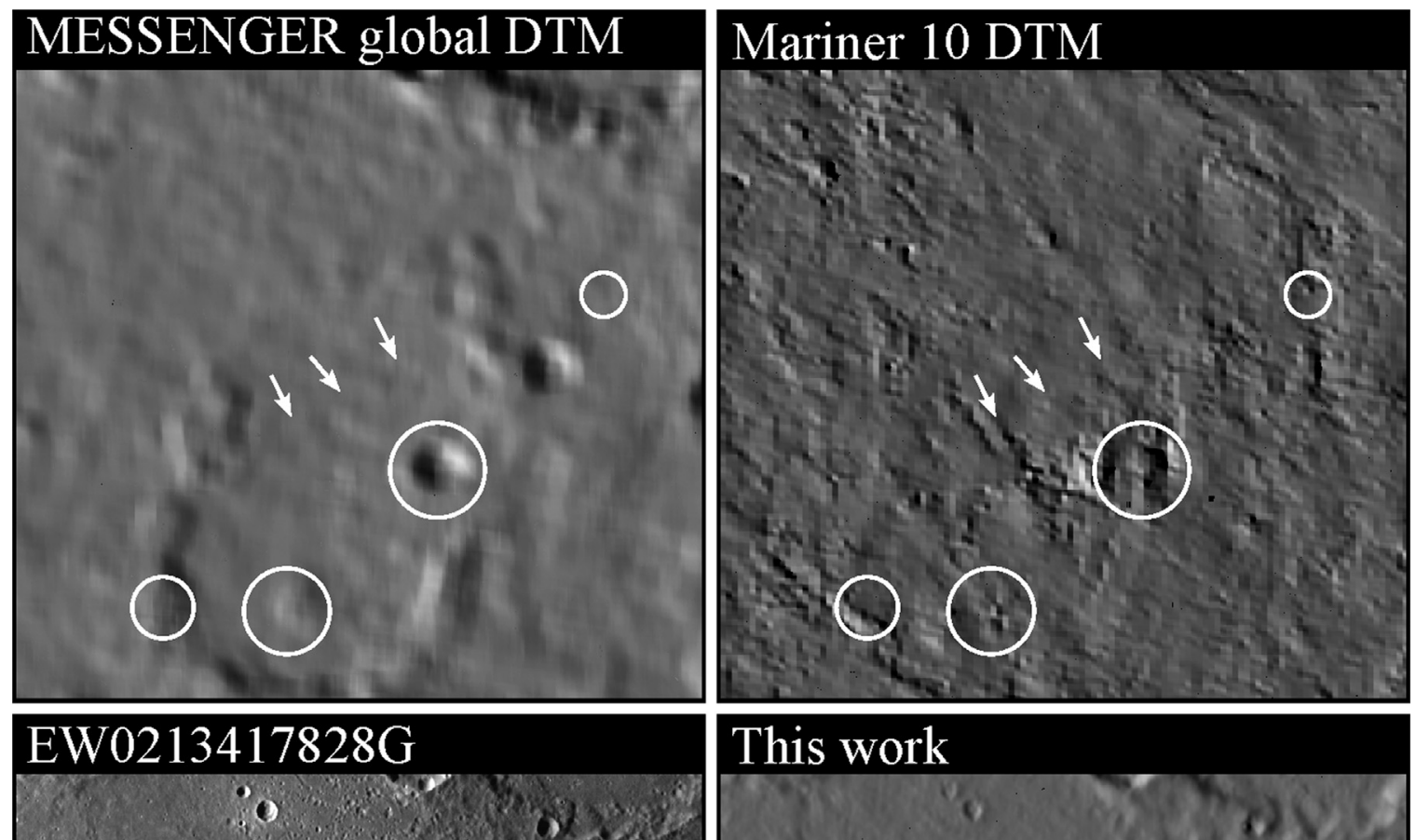

)
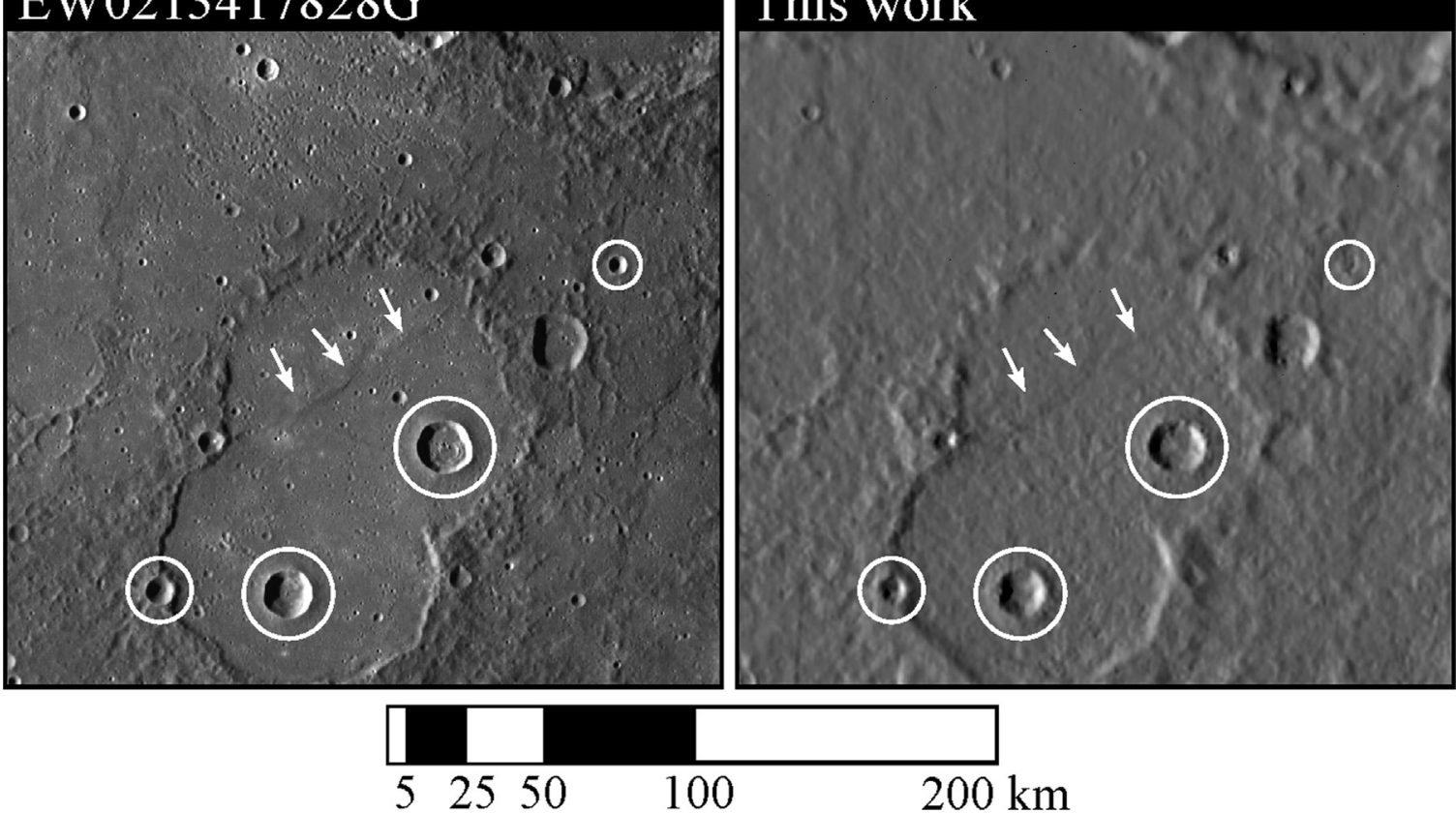

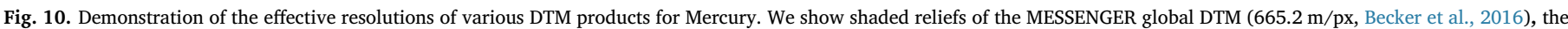

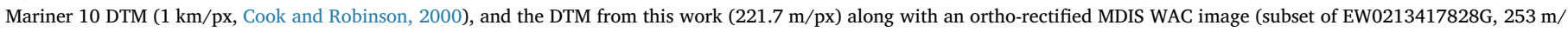

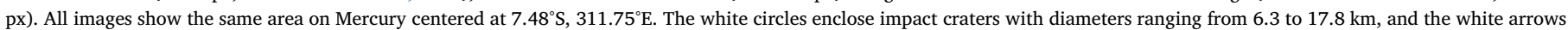

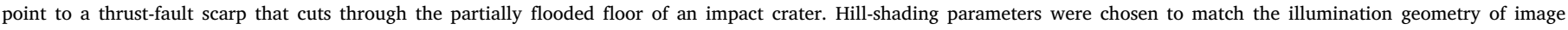
EW0213417828G.

After co-registration, MLA and H6 DTM profiles show excellent agreement (e.g., Fig. 9). We find a root mean squared height difference of $88 \mathrm{~m}$, in agreement with the estimated formal accuracy of the stereo DTM. The $1.5 \mathrm{~km}$ deep pit within the Catullus crater (left part of Fig. 9) as well as smaller craters and topography variations are well reproduced in the stereo DTM and in the MLA measurements. When MLA approaches its ranging limits (right part of Fig. 9), data gaps and false detections become apparent.

\section{Discussion and summary}

The H6 DTM was computed as a prototype, to demonstrate techniques for the joint processing of the very large number of MESSENGER images that will be needed for a global DTM. Also, in this study, we carried out a comprehensive quality assessment of our data product.

By visual inspection of craters, we estimate that the DTM has an effective lateral resolution of $5 \mathrm{~km}$ (see Fig. 10), in agreement with earlier estimates from similar stereo terrain models (Preusker et al., 2011; Stark et al., 2015b). The effective resolution of a stereo DTM depends on image resolution, observation conditions, and in particular stereo geometry, and therefore varies accordingly within the DTM (see Figs. 1 and 2). The estimate based on the region in Fig. 10 is representative for most parts of the DTM and can be considered as an average. Comparisons reveal that our DTM has a significantly higher effective resolution than the global stereo DTM (Becker et al., 2016) (with its nominal ground pixel size of $665 \mathrm{~m}$ ) (see Fig. 10). Further, the H6 DTM greatly exceeds the resolution of previous Mariner-10-based DTM products (Cook and Robinson, 2010). On the basis of this comparison, we can conclude that the DTM obtained in this work 
a

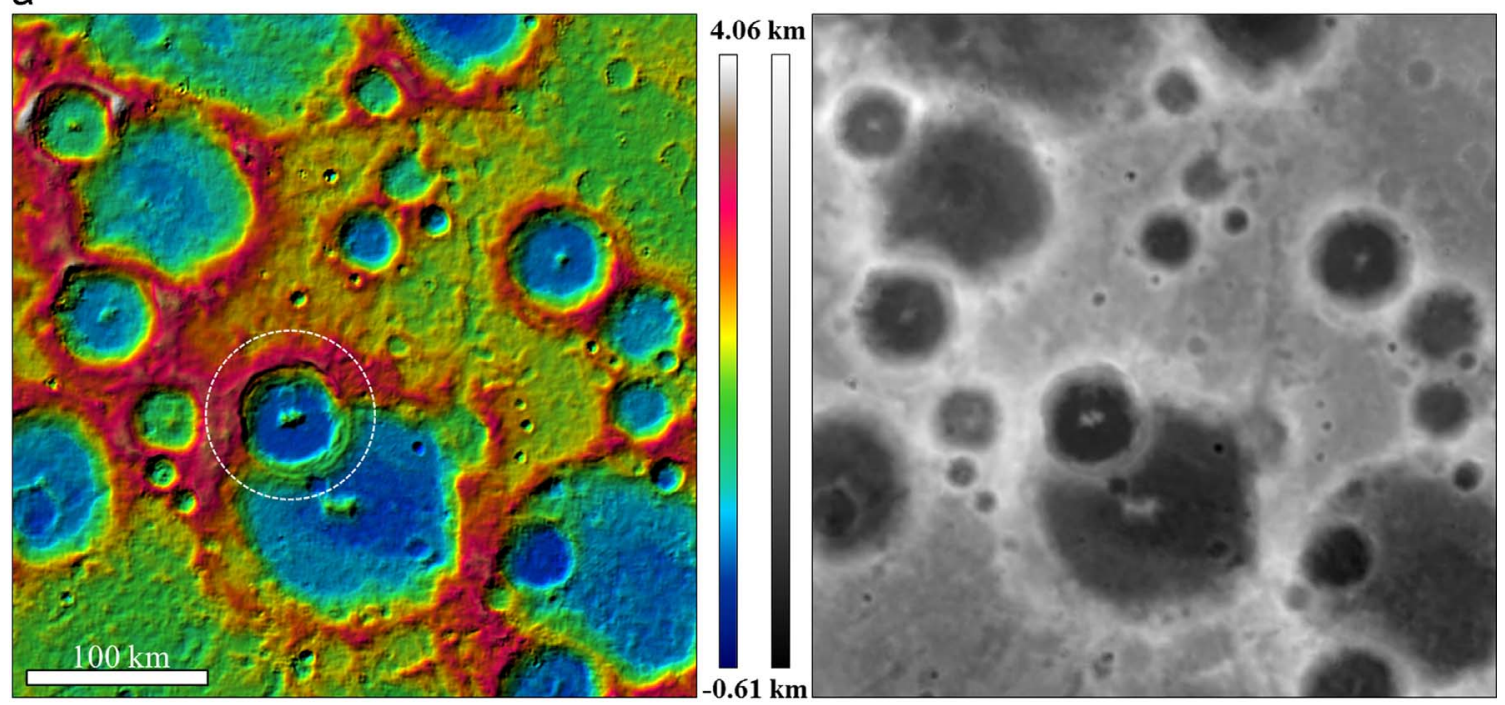

b

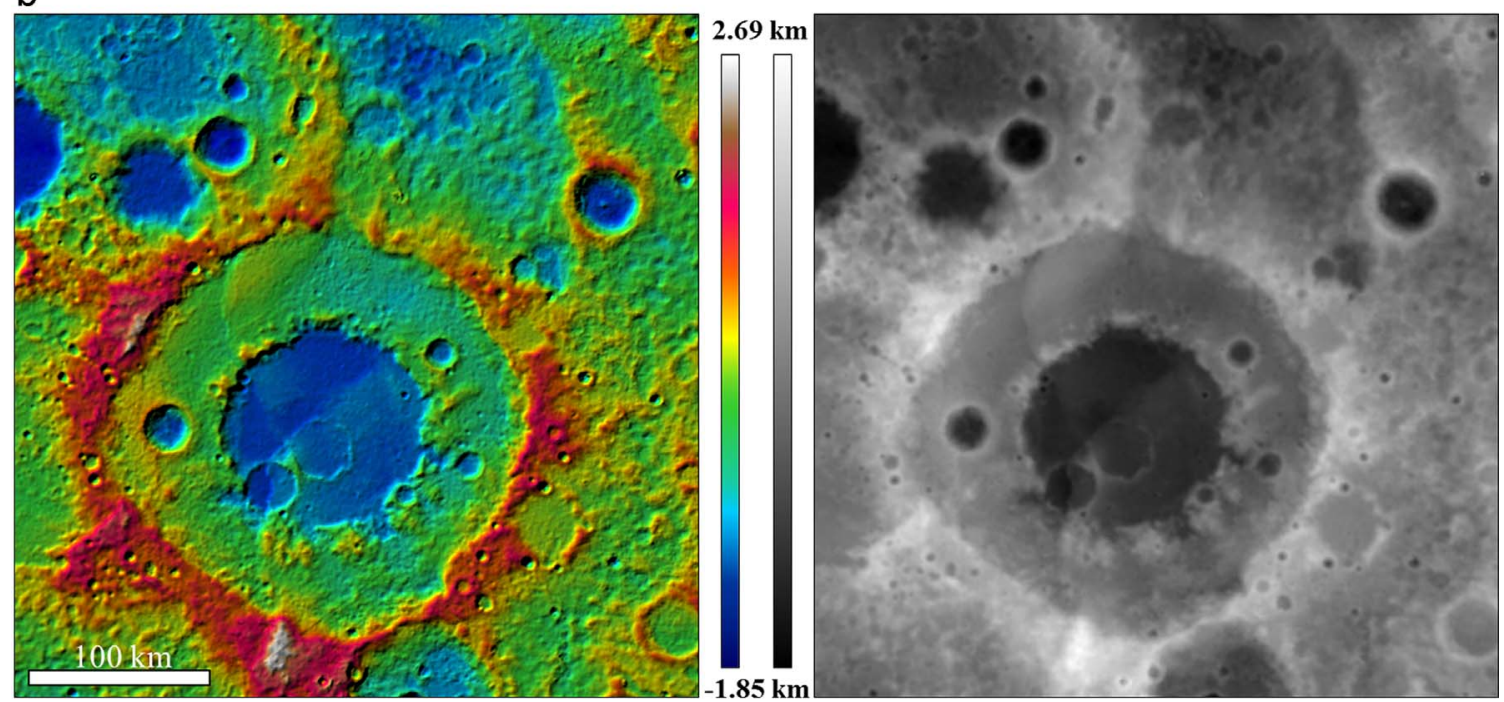

C

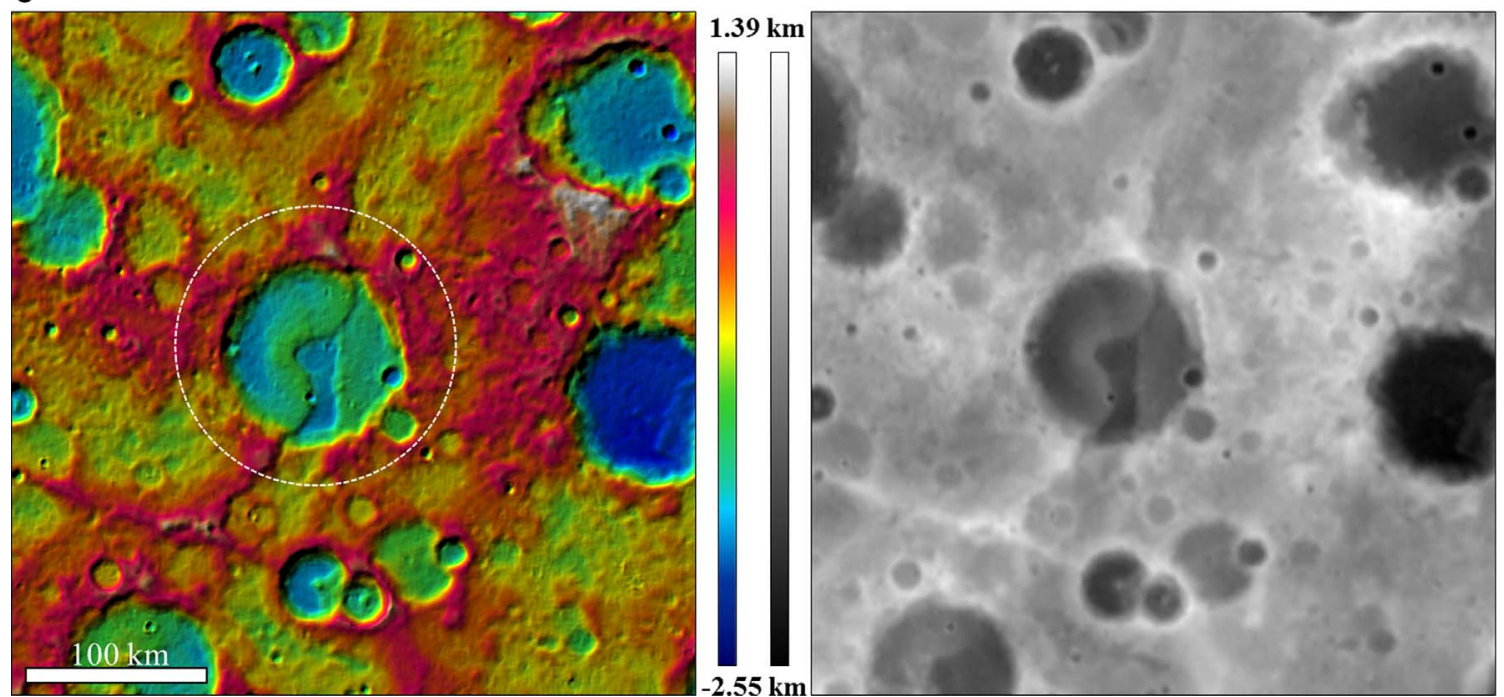

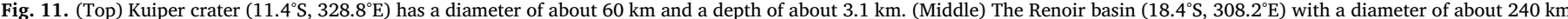

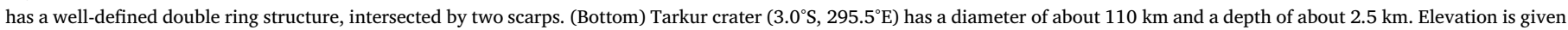
relative to the spherical datum as in Fig. 5. Elevations in the left column are shown in color on shaded relief; those in the right column are shown in grey scale. 

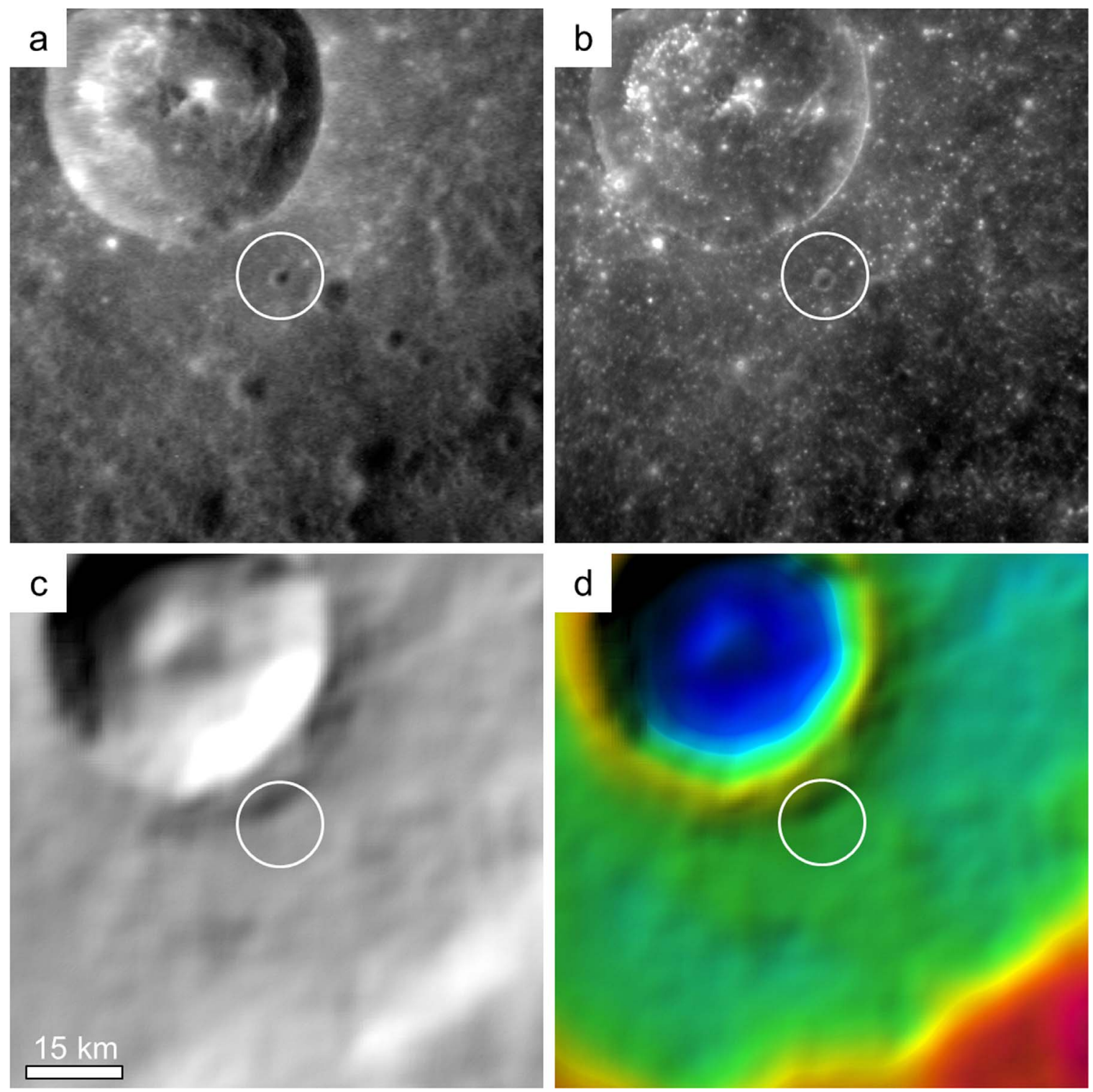

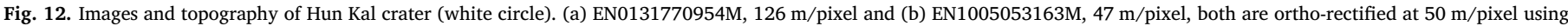
the H6 DTM. (c and d) show the corresponding area of the DTM, hill-shaded in grey scale and color-coded. The crater is visible in the images but cannot be resolved in the DTM.

Table 2

Measurements of the latitude and longitude of Hun Kal crater covered by five images.

\begin{tabular}{llll}
\hline Image & Latitude, ${ }^{\circ} \mathrm{N}$ & Longitude, ${ }^{\circ} \mathrm{E}$ & Image scale, m/pixel \\
\hline EN0131770954M & -0.463 & 339.996 & 126 \\
EN1002521325M & -0.465 & 339.996 & 54 \\
EN1004678277M & -0.466 & 339.994 & 134 \\
EN1004678315M & -0.465 & 339.993 & 134 \\
EN1005053163M & -0.467 & 339.995 & 47 \\
Average & -0.465 & 339.995 & \\
\hline
\end{tabular}

provides the best currently available topographic data set for the $\mathrm{H} 6$ Mercury quadrangle. Topographic landforms such as crater rims, pits, ejecta rays, and thrust-fault scarps are precisely reconstructed in the DTM and are suitable for detailed geological analysis.

The model features three well-known impact basins, Sanai $(490 \mathrm{~km})$, Homer $(314 \mathrm{~km})$, and Renoir $(246 \mathrm{~km})$, as listed in the catalog of Fassett et al. (2012), as well as an unnamed basin west of Homer (about $400 \mathrm{~km}$ diameter), for which detailed studies of morphology can be made (see Fig. 5). In addition, the model shows a multitude of craters (we estimate more than 50 craters larger than
$100 \mathrm{~km}$ in diameter), for which reliable measurements of depth-todiameter ratios are possible (Fig. 11).

Also found in the higher-elevation intercrater plains of the Kuiper quadrangle are lobate scarps, the surface expression of thrust faults formed as a result of global contraction (Solomon et al., 2008; Byrne et al., 2014; Watters et al., 2015). The most prominent is Santa Maria Rupes $\left(\sim 6^{\circ} \mathrm{N}, 340^{\circ} \mathrm{E}\right)$, a scarp with about $500 \mathrm{~m}$ of relief. Accurate topography of a lobate scarp is critical to constraining models of the geometry and depth extent of the associated thrust faults (EgeaGonzalez et al., 2012; Watters et al., 2016). Estimates of the maximum depth extent of a fault constrains the mechanical and thermal structure of Mercury's lithosphere at the time of faulting (Watters and Nimmo, 2010; Egea-Gonzalez et al., 2012).

Furthermore, the DTM can be used as a tool for the orthorectification and co-registration of MDIS images at resolutions of better than $200 \mathrm{~m}$ /pixel (Fig. 12). Among all processed images are two highresolution images $(50 \mathrm{~m} /$ pixel) and three images at $130 \mathrm{~m} /$ pixel that include the small Hun Kal crater, which defines Mercury's longitude system. Whereas the nominal location of Hun Kal is at $0.5^{\circ} \mathrm{S}$ and $340^{\circ} \mathrm{E}$, we measure the central location at $0.465^{\circ} \mathrm{S}, 339.995^{\circ} \mathrm{E}$ (see Table 2), attesting to the correct positioning of the DTM within $212 \mathrm{~m}$, i.e., 
approximately one DTM pixel (Fig. 12). While it is possible to shift the DTM to match the position of Hun Kal, no such effort has been made, in order to maintain the consistency of our DTM with other MESSENGER data products. Hence, the correct positioning of the DTM in Mercury's longitude and prime meridian system remains to be accomplished.

The DTM is found to be geometrically rigid, with minimal geometric distortions when compared with MLA data. The offsets that are observed between MLA profiles and the stereo DTM can be explained by errors in the reconstruction of the orbit and attitude of the spacecraft during acquisition of the MLA profiles and by errors in the rotational model of Mercury. However, the uncertainties within the Mercury rotation model are in the order of $200 \mathrm{~m}$ (Stark et al., 2015a) and cannot solely explain the observed offsets. Hence, the H6 DTM can be used as a reference to test the positioning and pointing of the MLA tracks or to determine unknown parameters of Mercury rotation, as was previously demonstrated with earlier DTM models (Stark et al., 2015a).

Considering the successful processing scheme developed in this paper, we currently prepare the production and delivery of DTMs for the other Mercury quadrangles (e.g. H3 (Preusker et al., 2017), H5, H7, and H10). We expect that individually produced neighboring DTMs will not match perfectly at their boundaries. Here we will benefit from images that connect more than one quadrangle or DTM to carry out a joint photogrammetric block adjustment for all involved images, which will solve lateral or vertical offsets at the DTM boundaries. Finally, we aim at the production of a high-resolution global DTM, which will require a final photogrammetric block adjustment of approximately 120,000 images from the 15 quadrangles. Such a product will allow more complete modeling of the MESSENGER orbit and Mercury rotation as well as comprehensive studies of Mercury's global morphology and tectonics.

The DTM described in this paper is available at the Planetary Data System (http://pdsimage.wr.usgs.gov/archive/mess-h-mdis-5-demelevation-v1.0/MESSDEM_1001/DEM/REGIONAL/IMG/MSGR_DEM_ DLR_EQ_H06_DM_222_I_V01.IMG).

\section{Acknowledgements}

Alexander Stark was funded by a grant from the German Research Foundation (OB124/11-1). Jürgen Oberst greatly acknowledges being hosted by MIIGAiK and supported by the Russian Science Foundation under Project 14-22-00197.

We thank MESSENGER's MLA and MDIS team members for their support, especially Susan Ensor, Haje Korth, Erwan Mazarico, Gregory Neumann, Michael Reid, and Sean Solomon.

\section{References}

Acton, C.H., 1996. Ancillary data services of NASA's navigation and ancillary information facility. Planet. Space Sci. 44, 65-70.

Albertz, J., Wiggenhagen, M., 2009. Guide for Photogrammetry and Remote Sensing. Wichmann Verlag, Heidelberg, Germany.

Archinal, B.A., et al., 2011. Report of the IAU working group on cartographic coordinates and rotational elements: 2009. Celest. Mech. Dyn. Astron. 109, 101-135. http://dx. doi.org/10.1007/s10569-010-9320-4.

Becker, K.J., et al., 2016. First global digital elevation model of mercury. Lunar Planet. Sci. 47, 2959. (abstract). 〈http://astrogeology.usgs.gov/search/map/Mercury/ Topography/MESSENGER/Mercury_Messenger_USGS_DEM_Global_665m $>$.

Byrne, P.K., et al., 2014. Mercury's global contraction much greater than earlier estimates. Nat. Geosci. 7, 301-307.

Cavanaugh, J.F., et al., 2007. The Mercury Laser Altimeter instrument for the MESSENGER mission. Space Sci. Rev. 131, 451-479. http://dx.doi.org/10.1007/ s11214-007-9273-4.

Cook, A.C., Robinson, M.S., 2000. Mariner 10 stereo image coverage of Mercury. J.
Geophys. Res. 105, 9429-9443.

Dermott, S.F., Thomas, P.C., 1988. The shape and internal structure of Mimas. Icarus 73, 25-65.

Egea-Gonzalez, I., et al., 2012. Depth of faulting and ancient heat flows in the Kuiper region of Mercury from lobate scarp topography. Planet. Space Sci. 60, 193-198.

Elgner, S., et al., 2014. Mercury's global shape and topography from MESSENGER limb images. Planet. Space Sci. 103, 299-308. http://dx.doi.org/10.1016/j.pss.2014.07. 019.

Fassett, C.I., et al., 2012. Large impact basins on Mercury: global distribution, characteristics, and modification history from MESSENGER orbital data. J. Geophys. Res. 117, E00L08. http://dx.doi.org/10.1029/2012JE004154.

Fjeldbo, G., et al., 1976. The occultation of Mariner 10 by Mercury. Icarus 29, 439-444.

Gläser, P., et al., 2013. Co-registration of laser altimeter tracks with digital terrain models and applications in planetary science. Planet. Space Sci. 89, 111-117.

Greeley, R., Batson, G., 1990. Planetary Mapping. Cambridge University Press.

Gwinner, K., et al., 2009. Derivation and validation of high-resolution digital terrain models from Mars Express HRSC-data. Photogramm. Eng. Remote Sens. 75, 1127-1142.

Hawkins III, S.E., et al., 2007. The Mercury Dual Imaging System on the MESSENGER spacecraft. Space Sci. Rev. 131, 247-338. http://dx.doi.org/10.1007/s11214-007. 9266-3.

Hawkins III, S.E., et al., 2009. In-flight performance of MESSENGER's Mercury Dual Imaging System. In: Hoover, R.B., Levin, G.V., Rozanov, A.Y., Retherford, K.D. (Eds.), SPIE Proceedings of Instruments and Methods for Astrobiology and Planetary Missions, Vol. 7441, paper 7441A-3. SPIE, Bellingham, Wash. p. 12.

Margot, J.L., et al., 2012. Mercury's moment of inertia from spin and gravity data. J. Geophys. Res. 117, E00L09. http://dx.doi.org/10.1029/2012JE004161.

Mazarico, E., et al., 2014. The gravity field, orientation, and ephemeris of Mercury from MESSENGER observations after three years in orbit. J. Geophys. Res. Planets 119, 2417-2436.

Oberst, J., et al., 2010. The morphology of Mercury's Caloris basin as seen in MESSENGER stereo topographic models. Icarus 209, 230-238.

Oberst, J., et al., 2011. Radius and limb topography of Mercury obtained from images acquired during the MESSENGER flybys. Planet. Space Sci. 59, 1918-1924.

Page, B.R., , et al., 2014. Tuning the MESSENGER state estimation filter for controlled descent to Mercury impact. Astrodynamics Specialist Conference, American Institute of Aeronautics and Astronautics/American Astronautical Society, paper AIAA-20144129, San Diego, CA. p. 16.

Perry, M.E., et al., 2011. Measurement of the radius of Mercury by radio occultation during the MESSENGER flybys. Planet. Space Sci. 59, 1925-1931.

Perry, M.E., et al., 2015. The low-degree shape of Mercury. Geophys. Res. Lett. 42, 6951-6958.

Preusker, F., et al., 2011. Stereo topographic models of Mercury after three MESSENGER flybys. Planet. Space Sci. 59, 1910-1917.

Preusker, F., et al., 2015. Shape model, reference system definition, and cartographic mapping standards for comet 67P/Churyumov-Gerasimenko - stereophotogrammetric analysis of Rosetta/OSIRIS image data. Astron. Astrophys. 583 (A33), 19.

Preusker, F., et al., 2017. High-resolution topography from MESSENGER orbital stereo imaging - The H3 quadrangle "Shakespeare". Lunar Planet. Sci. 48, 1441.

Scholten, F., et al., 2005. Mars Express HRSC data processing - Methods and operational aspects. Photogramm. Eng. Remote Sens. 71, 1143-1152.

Solomon, S.C., et al., 2001. The MESSENGER mission to Mercury: scientific objectives and implementation. Planet. Space Sci. 49, 1445-1465.

Solomon, S.C., et al., 2008. Return to Mercury: a global perspective on MESSENGER's first Mercury flyby. Science 321, 59-62.

Stark, A., et al., 2015a. First MESSENGER orbital observations of Mercury's librations. Geophys. Res. Lett. 42, 7881-7889.

Stark, A., et al., 2015b. Mercury's rotational parameters from MESSENGER image and laser altimeter data: a feasibility study. Planet. Space Sci. 117, 64-72.

Sun, X., Neumann, G.A., 2015. Calibration of the Mercury Laser Altimeter on the MESSENGER spacecraft. IEEE Trans. Geosci. Remote Sens. 53, 2860-2874.

Thomas, P.C., et al., 2007. Shapes of the Saturnian icy satellites and their significance. Icarus 190, 573-584.

Watters, T.R., Nimmo, F., 2010. The tectonics of Mercury. In: Watters, T.R., Schultz, R.A. (Eds.), Planetary Tectonics. Cambridge Univ. Press, New York, pp. 15-80.

Watters, T.R., et al., 2015. Distribution of large-scale contractional tectonic landforms on Mercury: implications for the origin of global stresses. Geophys. Res. Lett. 42, 3755-3763.

Watters, T.R., et al., 2016. Fault-bound valley associated with the Rembrandt Basin on Mercury. Geophys. Res. Lett. 43. http://dx.doi.org/10.1002/2016GL070205.

Wewel, F., 1996. Determination of conjugate points of stereoscopic three line scanner data of Mars 96 mission. Int. Arch. Photogramm. Remote Sens. 31, 936-939.

Zuber, M.T., et al., 2012. Topography of the northern hemisphere of Mercury from MESSENGER laser altimetry. Science 336, 217-220. http://dx.doi.org/10.1126/ science. 1218805 